DFPD01/TH/13

hep-th/0105041

\title{
THE INSTANTON UNIVERSAL MODULI SPACE OF N=2 SUPERSYMMETRIC YANG-MILLS THEORY
}

\author{
MARCO MATONE \\ Department of Physics "G. Galilei" - Istituto Nazionale di Fisica Nucleare \\ University of Padova \\ Via Marzolo, 8 - 35131 Padova, Italy \\ matone@pd.infn.it
}

\begin{abstract}
We use the recursive structure of the compactification of the instanton moduli space of $N=$ 2 Super Yang-Mills theory with gauge group $S U(2)$ to construct, by an inductive limit, a universal moduli space which includes all the multi-instanton moduli spaces. Furthermore, by exploiting an analogy with the strong and weak coupling expansions in the Matrix Model formulation of 2D Quantum Gravity, we discuss the possible field theoretical meaning of the contributions to the prepotential in the strong coupling region. In particular, whereas the weak and strong coupling expansions of the Painlevé I correspond to the genus and punctured spheres expansions, in the case of the instantons there should exist a space dual to the instanton moduli space.
\end{abstract}


Recently in [1] it has been shown that the instanton moduli spaces of $N=2$ Super Yang-Mills theory with gauge group $S U(2)$ admit a compactification which is at the heart of the recursive relations derived in [2]. The structure of this compactification explains why, in spite of the technical difficulties, the Seiberg-Witten (SW) solution [3] is simple. In the SW model there exists a relation between the modulus $u=\left\langle\operatorname{Tr} \phi^{2}\right\rangle$ and the effective prepotential [2] (see also [4), which allowed to prove the SW conjecture by using the reflection symmetry of quantum vacua [5]. Thus, essentially, the research started from the exact solution and ended with the reconstruction of the field theoretic structure of the theory.

A basic point in [1] was the observation of the strict similarity between the properties of the instanton moduli space and its measure on the one hand and the corresponding quantities in the theory of punctured spheres, on the other. In particular, it has been shown that a compactification similar to the one by Deligne-Knudsen-Mumford [6] and the restriction phenomenon satisfied by the Weil-Petersson volume form [7] lead to the recursive structure obtained in the SW model. The coefficients which determine the divisors at the boundary of the instanton moduli space and the structure of the instanton 2-form are fixed by the coefficients defining the recursion relations [2]. This mechanism is similar to the one considered in [8, 9]. In this respect the appearance of linear differential equations plays a crucial role [10, 1, 2, 11]. According to [11], this may be related to a sort of mirror phenomenon. In particular, we recall that the recursion relations satisfied by the instanton contributions and by the Weil-Petersson volume forms are fixed by an underlying linear differential equation. In [1] it has been shown that the instanton contribution to

$$
\left\langle\operatorname{Tr} \phi^{2}\right\rangle=a^{2} \sum_{n=0}^{\infty} \mathcal{G}_{n}\left(\frac{\Lambda}{a}\right)^{4 n}
$$

can be expressed as

$$
\mathcal{G}_{n}=\int_{\bar{V}_{I}^{(n)}} \bigwedge_{k=1}^{2 n-1} \omega_{I}^{(n)}
$$

The 2-form $\omega_{I}^{(n)}$ is defined on $\bar{V}_{I}^{(n)}$ denoting a suitable compactification of the $n$-instanton moduli space $V_{I}^{(n)}$. This result follows from some algebraic geometrical calculations whose details are given in [1]. Let us stress that the integral reproduces the recursion relations [2, [1]

$$
\mathcal{G}_{n+1}=\frac{1}{2(n+1)^{2}}\left[(2 n-1)(4 n-1) \mathcal{G}_{n}+\sum_{k=0}^{n-1} b_{k, n} \mathcal{G}_{n-k} \mathcal{G}_{k+1}-2 \sum_{j=1}^{n-1} \sum_{k=1}^{j} d_{j, k, n} \mathcal{G}_{n-j} \mathcal{G}_{j+1-k} \mathcal{G}_{k}\right]
$$

where $n>0, \mathcal{G}_{1}=1 / 4, b_{k, n}=c_{k, n}-2 d_{k, 0, n}$ and

$$
c_{k, n}=2 k(n-k-1)+n-1, \quad d_{j, k, n}=[2(n-j)-1][2 n-3 j-1+2 k(j-k+1)] .
$$


The above result is due to two basic properties. First, as we said, the compactification of $V_{I}^{(n)}$ is similar to the Deligne-Knudsen-Mumford compactification. Actually, this has an obvious recursive structure. In the case of instantons we have that the boundary of the $(2 n-1)$ dimensional space $\bar{V}_{I}^{(n)}$ decomposes as [1]

$$
\mathcal{D}^{(n+1)}=\bar{V}_{I}^{(n+1)} / V_{I}^{(n+1)}=\sum_{j=0}^{n-1} \mathcal{D}_{1, j}+\sum_{j=1}^{n-1} \sum_{k=1}^{j} \mathcal{D}_{2, j, k}+\mathcal{D}_{3, n}
$$

where

$$
\begin{aligned}
\mathcal{D}_{1, j} & =c_{n, j}^{(1)} \bar{V}_{I}^{(n-j)} \times \bar{V}_{I}^{(j+1)}, \\
\mathcal{D}_{2, j, k} & =c_{n, j, k}^{(2)} \bar{V}_{I}^{(n-j)} \times \bar{V}_{I}^{(j+1-k)} \times \bar{V}_{I}^{(k)} \times \bar{V}_{I}^{(1)} \\
\mathcal{D}_{3, n} & =c_{n}^{(3)} \bar{V}_{I}^{(n)} \times \bar{V}_{I}^{(1)} .
\end{aligned}
$$

Note that $\mathcal{D}_{3, n}$ can be included either in $\mathcal{D}_{1,0}$ or $\mathcal{D}_{1, n-1}$ by changing the coefficients. The second relevant property we used to reproduce the recursion relations is the restriction phenomenon. This is a property satisfied by the Weyl-Petersson 2-form. We defined $\omega_{I}^{(n)}$ in such a way that a similar phenomenon occurs also in the present framework. Roughly speaking, this phenomenon consists in the property that the restriction of $\omega_{I}^{(n)}$ to a component, e.g. to $\bar{V}_{I}^{(k)}$, of the boundary of $\bar{V}_{I}^{(n)}$, is in the same cohomological class of $\omega_{I}^{(k)}$. By simple calculations it is then easy to see that these two properties allow to reproduce the recursion relations. The parameters defining $\omega_{I}^{(n)}$ and $\bar{V}_{I}^{(n)}$ are fixed by the parameters of the recursion relations themselves. Let us then recall how $\omega_{I}^{(n)}$ is constructed. Let $\mathcal{D}_{\omega}^{(n+1)}$ be the $4 n$-cycle corresponding to the Poincaré dual to the "instanton" class $\left[\omega_{I}^{(n+1)}\right]=c_{1}\left(\left[\mathcal{D}_{\omega}^{(n+1)}\right]\right)$, where $[\mathcal{D}]$ is the line bundle associated to a given divisor $\mathcal{D}$ and $c_{1}$ denotes the first Chern class. In terms of the divisors at the boundary of the moduli space we have

$$
\mathcal{D}_{\omega}^{(n+1)}=\sum_{j=0}^{n-1} d_{n, j}^{(1)} \mathcal{D}_{1, j}+\sum_{j=1}^{n-1} \sum_{k=1}^{j} d_{n, j, k}^{(2)} \mathcal{D}_{2, j, k}+d_{n}^{(3)} \mathcal{D}_{3, n}
$$

The coefficients satisfy the relations [1]

$$
\begin{aligned}
& d_{n, k}^{(1)} c_{n, k}^{(1)}\left(\begin{array}{c}
2 n \\
2(n-k)-1
\end{array}\right)=\frac{b_{k, n}}{2(n+1)^{2}}, \\
& d_{n, j, k}^{(2)} c_{n, j, k}^{(2)}\left(\begin{array}{c}
2 n \\
2 k
\end{array}\right)\left(\begin{array}{c}
2(n-k) \\
2(n-j)-1
\end{array}\right)=-\frac{2 d_{j, k, n}}{k(n+1)^{2}}, \\
& d_{n}^{(3)} c_{n}^{(3)}=\frac{(2 n-1)(4 n-1)}{n(n+1)^{2}} .
\end{aligned}
$$


An open problem in SW theory consists in understanding the field theoretical characterization of the theory in the strong coupling region. In particular, it is not clear how it is possible to determine, by purely field theoretical means, the coefficients in the expansion of the prepotential near $u= \pm \Lambda^{2}$. Thus, it would be desirable to find expressions representing, in the strong coupling region, the analogues of the integrals on the instanton moduli spaces. Usually, the theory in the strong coupling region is investigated by considering the corresponding dual Abelian theory. However, the meaning of the coefficients in the expansion of $\mathcal{F}_{D}$ is still unclear. On the other hand, from a mathematical point of view, the expansion of $\mathcal{F}$ itself in the strong coupling region is well defined. In this context, we observe that the SW solution is essentially fixed by the transformation properties of the prepotential under duality. In other words, the transformation properties of the prepotential essentially fixes $\mathcal{F}$ itself. In turn, it determines all the instanton contributions. A possible way to investigate the dual space is to relate the two regions by deforming the instanton moduli space. In this way we should obtain the corresponding space near to $u= \pm \Lambda^{2}$. A natural approach is to consider the Borel sum. In particular, in the following we will express $u=\left\langle\operatorname{Tr} \phi^{2}\right\rangle$ as an integral on an infinite dimensional space which depends on a parameter on which we will perform the Borel sum. This is a first step towards expressing relevant quantities on spaces which, outside the weak coupling region, do not correspond to instanton moduli spaces. A similar expression for the prepotential $\mathcal{F}$ can be obtained from $u$ by using [2]

$$
u=\pi i\left(\mathcal{F}-a^{2} \frac{\partial \mathcal{F}}{\partial a^{2}}\right)
$$

which is equivalent to

$$
\mathcal{F}(a)=a^{2}\left(\frac{2 i}{\pi} \int_{a_{0}}^{a} d y y^{-3} \mathcal{G}(y)+\frac{a_{D 0}}{2 a_{0}}-\frac{i}{\pi} \frac{u_{0}}{a_{0}^{2}}\right),
$$

where $u=\Lambda^{2} \mathcal{G}(a)$, while $u_{0}$ and $a_{D 0}$ correspond to $u$ and $a_{D}$ at $a_{0}$.

Although we know the differential equation satisfied by $\left\langle\operatorname{Tr} \phi^{2}\right\rangle$ and $\mathcal{F}$, as we said, we are interested in deriving their expression outside the weak coupling region by assuming Borel summability. Thus, we will consider the expression

$$
\left\langle\operatorname{Tr} \phi^{2}\right\rangle=a^{2} \int_{0}^{\infty} d x e^{-x} \sum_{n=0}^{\infty} \frac{1}{n !} \mathcal{G}_{n} x^{n}\left(\frac{\Lambda}{a}\right)^{4 n},
$$

where $\mathcal{G}_{0}=1 / 2$. The precise domain of this Borel sum is related to an asymptotic analysis which we will consider elsewhere. One may also investigate other analytic continuations. We now define the "instanton universal moduli space" $\overline{\mathcal{V}}^{(\infty)}(q)$. Let us consider the embedding

$$
i_{n}: \bar{V}_{I}^{(n)} \longrightarrow \bar{V}_{I}^{(n+1)}, \quad n>1
$$


Let $q \in \mathbb{R}_{+}$, we define by inductive limit

$$
\overline{\mathcal{V}}^{(\infty)}(q)=\coprod_{n=0}^{\infty}\left(\bar{V}_{I}^{(n)} \times\left[0, q^{n}\right]\right) /\left(\bar{V}_{I}^{(n)}, q^{n}\right) \sim\left(i_{n} \bar{V}_{I}^{(n)}, 0\right)
$$

where $\bar{V}_{I}^{(0)}$ is a point. Let $d y$ denote the Lebesgue measure on the real axis and consider the indefinite rank forms

$$
\eta^{(\infty)}=\left[\frac{1}{2}+\sum_{n=1}^{\infty} \frac{\omega_{I}^{(n)^{2 n-1}}}{n !}\right] \wedge d y
$$

We then have

$$
\left\langle\operatorname{Tr} \phi^{2}\right\rangle=a^{2} \int_{0}^{\infty} d x e^{-x} \int_{\overline{\mathcal{V}}^{(\infty)}\left(x \Lambda^{4} / a^{4}\right)} \eta^{(\infty)}
$$

This expression should correspond to the one obtained by purely field theoretical means. This would provide a possible approach to understanding the role of the higher order contributions to $\mathcal{F}_{D}$. In this respect we observe that the moduli space in (15) depends on $x$. We note that, in principle, similar moduli spaces in the strong coupling region can be constructed by using the recursion relations which follow by expanding the prepotential at $u= \pm \Lambda^{2}$.

In the case in which $a \rightarrow \infty$, Eq.(15) reproduces the usual result in the form of an integral on the infinite-dimensional universal moduli space. In particular, we have

$$
\left\langle\operatorname{Tr} \phi^{2}\right\rangle=a^{2} \int_{\overline{\mathcal{V}}^{(\infty)}\left(x \Lambda^{4} / a^{4}\right)} \omega^{(\infty)}
$$

where

$$
\omega^{(\infty)}=\left[\frac{1}{2}+\sum_{n=1}^{\infty} \omega_{I}^{(n)^{2 n-1}}\right] \wedge d y .
$$

We observe that the appearance of a recursive structure similar to the one considered in the Deligne-Knudsen-Mumford compactification suggests a deep relation between the geometry of 4D SYM theory and the theory of Riemann surfaces. On the other hand, we already encountered a similar structure when the role of uniformization (Liouville) theory appeared to be relevant in the SW solution [2, 5].

The similarity between this approach and the theory of moduli space of punctured spheres suggests another analogy. Namely, in the Matrix Model formulation of 2D Quantum Gravity, it has been shown that the theory is described by the Painlevé I equation. In particular, in [9] it has been argued that, while the genus expansion corresponds to the weak coupling expansion, the expansion labeled by the number of punctures inserted on the sphere corresponds to a strong coupling expansion. This similarity, and the above remarks, suggest that the expansion at strong coupling corresponds to a new kind of moduli space, which is a sort of dual of the instanton moduli space. Presumably, this investigation is related to the Seiberg-Witten monopole equation [12]. 
In conclusion, we have introduced the instanton universal moduli space for $N=2 \mathrm{SYM}$ theory with gauge group $S U(2)$. In particular, we used the recursive structure of the compactification of instanton moduli space introduced in [1] to build, by an inductive limit, an infinite-dimensional moduli space which encompasses all instanton moduli spaces.

Acknowledgements. We would like to thank D. Bellisai and J. Isidro for many fruitful discussions. Work partly supported by the European Commission TMR programme ERBFMRXCT96-0045.

\section{References}

[1] M. Matone, JHEP 0104 (2001) 041, hep-th/0103246.

[2] M. Matone, Phys. Lett. B357 (1995) 342; Phys. Rev. D53 (1996) 7354.

[3] N. Seiberg and E. Witten, Nucl. Phys. B426 (1994) 19; ibid. B431 (1994) 484.

[4] J. Sonnenschein, S. Theisen and S. Yankielowicz, Phys. Lett. 367 (1996) 145. T. Eguchi and S.-K. Yang, Mod. Phys. Lett. A11 (1996) 131. G. Bonelli and M. Matone, Phys. Rev. Lett. 76 (1996) 4107; ibid. 77 (1996) 4712. F. Fucito and G. Travaglini, Phys. Rev. D55 (1997) 1099. N. Dorey, V. V. Khoze and M. P. Mattis, Phys. Lett. B390 (1997) 205. P. S. Howe and P. C. West, Nucl. Phys. B486 (1997) 425. E. D'Hoker, I. M. Krichever and D. H. Phong, Nucl. Phys. B494 (1997) 89. J.D. Edelstein, M. Marino and J. Mas, Nucl. Phys. B541 (1999) 671. G. Chan and E. D'Hoker, Nucl. Phys. 564 (2000) 503. J.D. Edelstein, M. Gomez-Reino and J. Mas, Nucl. Phys. B561 (1999) 273. J.D. Edelstein, M. Gomez-Reino, M. Marino and J. Mas, Nucl. Phys. B574 (2000) 587.

[5] G. Bonelli, M. Matone and M. Tonin, Phys. Rev. D55 (1997) 6466.

[6] P. Deligne and D. Mumford, IHES Publ. Math. 36 (1969) 75. F. Knudsen and D. Mumford, Math. Scand. 39 (1976) 19. F. Knudsen, Math. Scand. 52 (1983) 200.

[7] S.A. Wolpert, Ann. of Math. 118 (1983) 491; Amer. J. Math. 107 (1985) 969.

[8] M. Matone, J. Geom. Phys. 21 (1997) 381.

[9] G. Bonelli, P.A. Marchetti and M. Matone, Phys. Lett. B339 (1994) 49; Lett. Math. Phys. 36 (1996) 189.

[10] A. Klemm, W. Lerche and S. Theisen, Int. J. Mod. Phys. A11 (1996) 1929. 
[11] M. Kontsevich and Yu. Manin (with Appendix by R. Kaufmann), Inv. Math. 124 (1996) 313. R. Kaufmann, Yu. Manin and D. Zagier, Comm. Math. Phys. 181 (1996) 763.

[12] E. Witten, Math. Res. Lett. 1 (1994) 769. G. Moore and E. Witten, Adv. Theor. Math. Phys. 1 (1998) 298. 